\title{
Présentation. La linguistique « hors du temple »
}

\section{Guy Achard-Bayle et Marie-Anne Paveau}

\section{OpenEdition}

\section{Journals}

Édition électronique

URL : http://journals.openedition.org/pratiques/1171

DOI : 10.4000/pratiques. 1171

ISSN : 2425-2042

\section{Éditeur}

Centre de recherche sur les médiations (CREM)

\section{Édition imprimée}

Date de publication : 15 décembre 2008

Pagination : 3-16

\section{Référence électronique}

Guy Achard-Bayle et Marie-Anne Paveau, «Présentation. La linguistique « hors du temple » », Pratiques [En ligne], 139-140 | 2008, mis en ligne le 15 décembre 2008, consulté le 08 mars 2021. URL : http:// journals.openedition.org/pratiques/1171; DOI : https://doi.org/10.4000/pratiques.1171 


\section{La linguistique « hors du temple »(1)}

\section{Pourquoi ce numéro ? ${ }^{(2)}$}

En même temps que cette livraison de la revue Pratiques, parait un numéro de l'European Review of Philosophy consacré à la Folk Epistemology (Heintz et al. (eds.) 2008) ${ }^{(3)}$. Parmi les questions abordées par les auteurs, majoritairement philosophes, certaines recouvrent exactement celles que nous posons dans notre numéro, preuve qu'il existe actuellement, en philosophie, en épistémologie, en linguistique mais aussi en psychologie, en argumentation, en neurosciences, en biologie et dans bien d'autres domaines, on y reviendra, une interrogation parfois musclée sur la nature des savoirs et sur les modes de constitution et de légitimation des connaissances dites scientifiques. L'European Review of Philosophy pose entre autres les interrogations suivantes :

- What is the proper domain of a folk epistemology system ?

- Do epistemic evaluations involve conscious thinking?

- Are epistemic evaluations human-specific ?

- How does folk epistemology contribute to rational thinking?

- What are the relations (if any) between normative epistemology, commonsense epistemology and folk epistemology? How does folk epistemology relate to our naive understanding of truth?

- Which aspects of culture could be explained on the basis of a folk epistemology?

- Do subjects share the same epistemological intuitions across cultures ? Or do epistemologies vary across cultures? (Heintz et al. 2008 : call for papers)

(1) L'expression «hors du temple», définition étymologique du mot profane, est empruntée au projet qu'Amélie Cure avait préparé pour ce numéro (voir Achard-Bayle ici même). Elle fait écho à la formule «hors les murs », qui désigne parfois l'art brut, l'art des fous, des enfants, des naïfs et des non-artistes, appelé parfois « art outsider » ou «folk art»...

(2) Ce texte adopte les rectifications orthographiques proposées dans le Journal officiel du 6.12.1990.

(3) Nous revenons plus bas en détail sur la dénomination folk et ses traductions en français et dans d'autres langues. 
La place nous manque ici pour expliquer l'émergence actuelle de ces interrogations. Nous nous contenterons de rappeler quelques phénomènes bien connus : l'augmentation du niveau de connaissances des individus dû en particulier au développement des nouvelles technologies, la disponibilité accrue des savoirs dans les publications traditionnelles ou électroniques, la surdiffusion de l'information sur des supports multiples, en particulier gratuits, tous ces phénomènes (qui concernent, nous ne l'ignorons pas, surtout les pays développés), contribuent à l'augmentation des savoirs des individus, et donc à un certain effacement des différences entre professionnels du savoir (que sont les universitaires par exemple) et détenteurs profanes de savoirs ou de savoirs profanes ${ }^{(4)}$. D'autres éléments plus confidentiels car ils concernent l'histoire des sciences peuvent être avancés : la réflexion sur les relations entre savoir et croyance est aussi vieille que la pensée elle-même, et partant, sur la validité des savoirs de sens commun non susceptibles de vérification logique (Coates 1996, Dascal 1999, Markovits 1999, Dennett 1990 [1987], Fisette \& Poirier 2002) ; l'émergence d'une discipline comme l'ethnométhodologie a amené dans la seconde moitié du $20^{\mathrm{e}}$ siècle de nouveaux objets épistémologiques pour les sciences humaines comme le « savoir des membres », ou de nouvelles méthodes comme la « compréhension », en particulier à partir des récits de vie ; enfin les sciences cognitives, contrairement à leur sulfureuse réputation positiviste et naturaliste, ont été les premières à se demander comment les connaissances étaient produites dans le cerveau... de tout un chacun, et non pas des seuls scientifiques. Il nous a donc semblé pertinent, et même relativement urgent, que la linguistique française, restée jusqu'à présent un peu à l'écart de ce questionnement épistémologique, s'interroge et se laisse interroger par la dimension folk des savoirs.

En posant, au sens propre, comme l'indique notre titre interrogatif (interrogateur?), la question de la linguistique populaire aux sciences du langage, nous poursuivons plusieurs objectifs. Nous souhaitons d'abord rendre compte de l'absence en France d'un champ identifié comme « la linguistique populaire » ou « la linguistique folk », par rapport aux domaines anglo-saxon et allemand, qui comptent parmi les sous-domaines de leur linguistique une folk linguistics ou une Volklinguistik assez bien implantée et dynamique, si l'on en croit les publications et les manifestations scientifiques repérables ces dernières années (voir la bibliographie). Nous voulons également explorer voire définir ce qui pourrait relever du champ de la linguistique populaire dans le domaine francophone, par rapport à des domaines connexes et des problématiques affines comme le purisme, la grammaire normative, les travaux sur les normes, sur le métalangage ou la notion d'épilinguistique par exemple. Nous voulons également ouvrir une réflexion sur la validité de savoirs profanes, et, par conséquent, sur celle des savoirs « scientifiques », question qui est très spécifique en linguistique, dans la mesure où la réflexivité, l'introspection, la conscience linguistique et épilinguistique sont des données définitoires de la discipline. Que valent donc les intuitions des locuteurs profanes par rapport aux élaborations savantes des linguistes ? Ces derniers ne sont-ils pas aussi des locuteurs profanes ? Il nous semble enfin nécessaire de questionner les relations et les apports de la linguistique populaire à la linguistique générale et appliquée pour la connaissance

(4) Cette distinction n'est pas binaire mais scalaire : de nombreuses catégories de détenteurs de savoirs peuvent être envisagées comme les experts, les étudiants, les collectionneurs, les passionnés, les érudits ou les spécialistes de tous ordres. Pour une analyse de ce type d'échelle, voir Schmale et Paveau ici même. 
et l'enseignement-apprentissage de la langue, en particulier à une période où les orientations officielles de l'enseignement de la langue à l'école et au collège promeuvent un type de grammaire qui revendique une "simplicité » et une « authenticité » peut-être proches de ce que nous appelons ici savoirs profanes ou spontanés ${ }^{(5)}$. C'est la raison pour laquelle nous avons souhaité une réflexion sur la place, la validité et l'efficacité pédagogique des pratiques relevant plus ou moins consciemment de la linguistique populaire, mises en œuvre par les élèves comme par leurs enseignants, dans l'enseignement de la langue à tous ses niveaux.

\section{Qu'est-ce que la linguistique populaire?}

Nous revenons ici sur des problèmes de désignation et de catégorisation des objets ou des questions relatifs à la linguistique populaire.

\subsection{Les termes et catégories de folk et populaire}

Le terme linguistique populaire est un calque d'une série de dénominations anglo-saxonnes basées sur folk, dans lesquelles folk est traduit en français par populaire, spontané, naïf, profane ou ordinaire (la liste restant ouverte, comme le montrent les articles de ce numéro). On parle aussi de linguistique de sens commun et l'on rencontre également l'expression linguistique des profanes, dont L. Rosier signale la présence désormais massive sur l'internet : «On peut [...] ajouter ce qu'on nomme "la linguistique des profanes", particulièrement visible sur l'internet, notamment dans le cadre des forums de discussion [...] 》 (Rosier 2004 : $70)$.

Dans le domaine scientifique anglo-saxon (surtout américain), on trouve des études assez nombreuses sur les folk theories : folk psychology, folk epistemology, folk biology (dont folk taxonomy), folk linguistics (dont folk etymology et folk ou perceptual dialectology) et récemment folk pragmatics. Le terme folk n'est cependant pas univoque et le domaine de la folk psychology (pour la diffusion en français des travaux américains voir par exemple Fisette \& Poirier 2002) se subdivise par exemple en deux sous-domaines : d'une part la psychologie de sens commun (commonsense psychology) qui décrit et explique le comportement humain en termes de croyances, désirs, intentions, attentes, espoirs, etc. ; d'autre part une version philosophique de cette psychologie de sens commun qui fournit une interprétation de ces explications ordinaires à travers des généralisations théoriques mettant en œuvre les concepts de croyance, désir, etc. (courant représenté par exemple par P. Churchland, D. Dennett, W. Sellars).

Les lexies construites sur folk étant bien stabilisées dans le champ anglo-saxon, nous conservons sa traduction la plus littérale malgré la polysémie de populaire en français : nous parlerons donc ici de linguistique populaire ${ }^{(6)}$. Mais comment définir ce terme? On proposera pour l'instant d'appeler « populaire» le savoir spontané

(5) Voir en particulier les textes suivants : Bulletin officiel $\mathrm{N}^{\circ} 0,20$ févr. 2008, Les nouveaux programmes de l'école primaire, Projet soumis à consultation, hors-série ; Éduscol dgesco, avril 2008, Collège, Projet de programme, français ; Rapport de mission sur l'enseignement de la grammaire, 2006, par A. Bentolila, E. Orsenna, D. Desmarchelier.

(6) Les réserves autour de ce terme réputé ambigu et trompeur (il désignerait de manière péjorative la linguistique du peuple) ont cependant amené certains auteurs à adopter la lexie francisée folk linguistique (voir Paveau ici même). 
des acteurs sur le monde (déposé dans les proverbes ou dictons par exemple), qui se distingue du savoir savant ou scientifique comme le savoir-faire se distingue du " savoir que » et le sens commun du savoir scientifique. Ce savoir spontané est constitué de connaissances empiriques, non susceptibles de vérification logique (le savoir spontané n'est ni vrai ni faux, on parle alors de « savoir approximatif», comme l'explique F. Markovits) et de croyances qui constituent des guides pour l'action: les légendes urbaines ou les influences de la lune sur la pousse des plantes sont des croyances relevant du savoir spontané. Les connotations péjoratives généralement attachées à ce populaire-là (contrairement à d'autres usages mélioratifs du mot comme dans éducation ou université ou arts et traditions populaire(s) par exemple) seront pour nous bien évidemment écartées et constitueront un des objets de recherche possibles pour ce domaine.

\subsection{Géographie de la linguistique populaire}

\section{- Folk linguistics}

Le domaine anglo-saxon est ouvert dans les années 1960 par les travaux de Hoenigswald $(1960,1966)$ qui réclame fermement la prise en compte des savoirs spontanés dans la constitution de toute science, sans que sa demande ne rencontre beaucoup d'échos à l'époque. Il faut attendre 2000 pour que l'ouvrage de N. Niedzielski et D. Preston propose une synthèse dont le contenu fournit en quelque sorte une image de la linguistique populaire américaine actuellement. Les auteurs précisent d'abord le sens de folk dans folk linguistics :

[The word folk] refers to those who are not trained professionals in the area under investigation [...] We definitely do not use folk to refer to rustic, ignorant, uneducated, backward, primitive, minority, isolated, marginalized, or lower status groups or individuals (2000: viii).

Ils signalent ensuite que la prise en compte des savoirs populaires contribue à la constitution du corps de savoirs scientifiques :

Whether for the purely scientific purpose of recording it or for the social purposes of helping us better understand our attitudes to one another, we suggest that the study of $[\ldots]$ linguistically-oriented caricature is an important task $(2000: 123)$.

Ensuite, les intitulés des quatre chapitres de leur ouvrage dessinent clairement la carte de la folk linguistics américaine :

1. Regionalism

2. Social Factors

3. Language Acquisition and Applied Linguistics

4. General and Descriptive Linguistics

Ce qui permet de remarquer avec J.R. Dow (qui fait le compte rendu du livre de N. Niedzielski et D. Preston dans le Journal of American Folklore en 2001) que l'ouvrage apporte des éléments d'analyse et de réflexion aux domaines suivants de la recherche linguistique américaine, dont les intitulés n'ont pas toujours de correspondant exact en français (nous les laissons alors tels quels) : the ethnography of language, the social psychology of language (ou language attitude study parfois traduit par psychologie sociale du langage), general, 
descriptive linguistics (linguistique générale, descriptive), language variation and change (variationnisme), et applied linguistics (linguistique appliquée). On comprend donc que, dans le domaine anglo-saxon en tout cas, la folk linguistics constitue un champ et un enjeu important pour les sciences du langage.

\section{- Volkslinguistik, Laienlinguistik}

Dans le domaine germanique, la Volkslinguistik, parfois appelée Laienlinguistik bien que les termes ne recouvrent pas les mêmes pratiques linguistiques (voir Schmale et Stegu ici même, ainsi que Antos 1996), qui résulte de la folk linguistics américaine comme le montre Brekle 1989, constitue également un domaine de recherche installé. G. Antos a largement contribué à faire connaitre cette approche, qui se situe plutôt dans le domaine de la formation, voire de la psychologie ou de la gestion : la Laienlinguistik, c'est la linguistique des manuels de conversation ou d'expression orale, destinée à améliorer les compétences langagières des locuteurs dans leur vie sociale et professionnelle. Les travaux de ce que l'on nomme plutôt Volklinguistikse concentrent plutôt sur la dialectologie et adoptent une perspective qu'on peut appeler géolinguistique, se portant par exemple sur l'image de "bad language » associée aux variantes régionales (voir par exemple Langer 2001, les travaux de W. Davies et le projet "The history and current status of 'bad language' as a concept in German folk linguistics » sur le site http ://eis.bris.ac.uk/ gexnl/research/ahrb.html). C'est également cet usage géolinguistique de la notion de linguistique spontanée que fait en Suisse P. Sériot, par exemple dans le titre de son article de 1996 sur la linguistique spontanée des traceurs de frontières et dans le travail collectif publié ici.

\section{- Linguistique populaire}

Dans le domaine français et francophone, les études se réclamant de la linguistique populaire sont bien rares, comme le signale J.-C. Beacco, qui choisit d'ailleurs d'éviter le terme populaire dans l'intitulé du numéro 154 de Langages qu'il dirige sur le sujet en 2004 (Beacco (dir.) 2004), préférant parler de «représentations métalinguistiques ordinaires » (il reconduit d'ailleurs sa réserve dans sa participation au présent numéro). C'est surtout le terme étymologie populaire qui est répandu en France, sans doute parce qu'il nomme un phénomène linguistique descriptible et susceptible de fournir un objet discret pour la linguistique. Outre l'article de H.E. Brekle dans le tome 1 de l'Histoire des idées linguistiques dirigé par S. Auroux, et le travail récent de J.-C. Beacco cité plus haut, on trouve des mentions explicites du domaine de la linguistique populaire chez P. Bourdieu, qui réclame dès 1983 une "science rigoureuse de la sociolinguistique spontanée » (Bourdieu 2001 : 137) et de manière plus récente et programmatique chez M.-A. Paveau, sur la prise en compte de la linguistique populaire dans l'enseignement de la langue (2005), sur la construction des objets sociaux dans les approches sociolinguistiques $(2007,2008)$ et dans le renouvellement des théories du discours (2006), ainsi que chez L. Rosier, à propos de la prise en compte nécessaire du purisme, forme de linguistique populaire, dans les travaux sur les normes de la langue et la circulation des discours $(2003,2004$, 2006).

Cela ne veut évidemment pas dire que les questions concernées ne soient pas 
traitées dans la littérature, mais elles le sont autrement, et sous d'autres étiquettes terminologiques ou d'autres orientations théoriques et thématiques.

D'abord, c'est sous l'appellation ordinaire que le discours spontané sur la langue est parfois étudié (voir par exemple le sommaire de Beacco (dir.) 2004), sans que la question ait jamais débouché en France sur la constitution d'un domaine scientifique visant à une certaine systématisation (la « linguistique ordinaire » ou « de l'ordinaire »?). Le terme ordinaire étant largement mobilisé par des lexies comme français ordinaire, échanges ordinaires ou discours ordinaires, la problématique de l'ordinaire se confond souvent avec celle du quotidien à la manière des ethnométhodologues. Ensuite, deux sous-domaines ou orientations théoriques et thématiques sont assez intensément traversés par les questions de la linguistique populaire (vu l'importance des champs concernés les références données ici ne sont que modestement indicatives et exemplificatrices) :

- d'une part le très vaste domaine du " méta », qu'il s'agisse de la linguistique du système (Rey-Debove 1978 qui distingue " métalangage courant 》 et « scientifique »), de la théorie de l'énonciation et de la reformulation (Authier 1995 et les boucles méta-énonciatives, Julia 2001 qui parle de « sémantique spontanée ») ou de la didactique de la langue (par exemple J.-C. Beacco, R. Bouchard, J. David, S. Trévise ; voir Bouchard, Meyer 1995 pour une synthèse);

- d'autre part l'ensemble tout aussi vaste des travaux qui, en sociolinguistique (J.-C. Beacco définit d'ailleurs la linguistique populaire comme une branche de la sociolinguistique), s'occupe des normes et des représentations (Berrendonner 1982, Gadet 2007 [2003], Houdebine 2002, Lafontaine 1986 et A. Boyer, N. Gueunier, J.-M. Klinkenberg et bien d'autres), parfois sous l'étiquette des « attitudes linguistiques » dans le cadre de la psychologie sociale du langage.

\subsection{Des pratiques linguistiques profanes}

Comme nous venons de montrer que, d'une certaine manière, la linguistique populaire n'existe pas en France, en tout cas comme champ constitué, il peut sembler paradoxal de vouloir en définir les objets et les matériaux d'observation. Le paradoxe tombe si l'on propose d'envisager la linguistique populaire comme un cadre d'unification des objets et approches évoqués jusqu'à présent, centré autour de la notion de pratique.

On fera à cette fin la proposition suivante : le domaine d'investigation de la linguistique populaire comprendrait 1'ensemble des énoncés que 1'on peut qualifier de pratiques linguistiques profanes (c'est-à-dire qui ne proviennent pas des représentants de la linguistique comme discipline établie, les «non-linguists » comme les appellent N. Niedzielski et D. Preston), désignant, évaluant ou se référant à des phénomènes langagiers pour produire (les deux premiers items sont repris de Brekle 1989 et le troisième proposé par nous) :

- des descriptions ou (pré)théorisations linguistiques, par exemple celles qui portent sur la désignation (appeler un chat un chat ou les choses par leur nom) ou sur la hiérarchie entre écrit et oral (se payer de paroles, paroles verbales, parler comme un livre) ou encore sur la conformité aux règles de la langue (malgré que et aller au coiffeur « ne sont pas français »). On citera comme exemple un extrait de linguistique populaire mondaine, issu du corpus des ouvrages typiques de «1'esprit français », qui propose une petite théorie profane du patronyme en 
contexte interculturel (Turid, la jeune compagne norvégienne du narrateur, fait l'expérience des usages du nom propre dans l'administration française) :

Mais le secret du nom s'étend aussi bien aux services publics. Alors que, dans de nombreux pays, on sait tout de suite à qui l'on a affaire - dans les bureaux grâce à un cartouche portant le nom du préposé ; au téléphone parce que le fonctionnaire s'est nommé - en France, si Turid veut savoir qui elle doit appeler à la préfecture, aux contributions, à la Sécurité sociale :

- Le poste 634 !

Si elle insiste pour avoir une interlocutrice qui lui a paru aimable :

- Demandez madame Yvette!

Le nom de famille reste un secret quasi inviolable. Il en est de même au bistrot où le patron reçoit sans cesse des messages codés :

- Je vous laisse ça pour Monsieur Léon. Vous lui direz que c'est de la part de Monsieur Raymond... (P. Daninos, 1977, Made in France, Paris, Julliard : 23)

- des prescriptions comportementales, qui relèvent la plupart du temps d'un normativisme plus ou moins exacerbé : on sait que les « amateurs de beau langage » ou « marchands de règles » selon 1'expression de G. Philippe (2002) condamnent facilement les néologismes (inutiles), les emprunts (menaçants), les adverbes en -ment (lourds), les mots techniques (jargonneux), les féminisations (ridicules), etc. (Paveau, Rosier 2008). Les pratiques profanes relèvent alors du purisme, ainsi défini par L. Rosier :

Il se caractérise par une forte axiologisation performative (ce qui se dit, ce qui ne se dit pas) qui rejoint le bon usage et entend respecter une stricte économie des échanges linguistiques, où on évalue celui qui parle selon sa maitrise de la langue, sous l'angle de la richesse lexicale et de la correction grammaticale (Rosier $2004: 69$ ).

- des interventions spontanées sur la langue, qualifiées de «fautes » par les grammairiens et puristes, mais qui constituent une véritable pratique linguistique profane implicite si l'on considère que la faute constitue un discours sur la langue. La chose est entendue pour les étymologies populaires (girouette < girer + pirouette) et les métanalyses ou « mécoupures » (omnibus $\rightarrow$ autobus, panorama $\rightarrow$ diaporama), moins en ce qui concerne les erreurs. Mais on sait pourtant, et depuis longtemps (l'indépassable Grammaire des fautes de Frei date de 1928) que la majorité des fautes des locuteurs petits ou grands s'expliquent par le principe d'économie de la langue : la langue est ainsi régularisée (le célèbre *vous faisez), simplifiée (le cas bien connu, honni des puristes, de émotion $\rightarrow$ émotionner ou moins connu de *il couvra, lu dans Télérama, hebdomadaire cultivé, en juillet 2006), harmonisée (*Centre d'études spaciales, également dans Télérama en septembre 2006). Les fautes de français existent-elles, comme le demande D. Leeman (1994) ? Tout dépend par qui, pour qui et pour quoi faire. Mais les interventions concernent également des champs plus politiques, qui concernent les realia des territoires et des populations, comme le montre bien ici l'article de P. Sériot, E. Bulgakova, A. Herzen. Noms des gens, noms des langues, noms des pays et des fleuves : se joue là une folk linguistique à fort degré de performativité puisque les interventions linguistiques sont aussi des prescriptions identitaires.

Pour récapituler, on dira que la linguistique populaire rassemble selon nous trois sortes de pratiques linguistiques : descriptives (on décrit l'activité de langage), normatives (on prescrit les comportements langagiers) et interventionnis- 
tes (on intervient sur les usages de la langue). Voyons maintenant, dans la perspective du numéro, quelles questions la linguistique populaire ainsi dessinée pose à la linguistique et à la didactique de la langue.

\section{Les dimensions multiples de la linguistique populaire}

Une des raisons pour lesquelles la linguistique populaire devrait constituer un domaine de la linguistique est qu'elle concerne non seulement la théorie linguistique dans sa constitution, sa validité et sa légitimité (ce qui lui donne une dimension épistémologique et métathéorique), mais également les pratiques langagières dans leurs dimensions sociales, culturelles et cognitives (ce qui la tire vers la sociolinguistique, la psychologie sociale et la sémantique cognitive), ainsi que les procédures d'enseignement-apprentissage de la langue (ce qui la mène vers la didactique de la langue et la psycholinguistique).

\section{1. Épistémologie}

La linguistique populaire pose un problème de frontières disciplinaires et de conception de la science. Sur ce point les questions se bousculent : quels sont les rapports entre sociolinguistique et linguistique populaire (intégration, affinité, croisement)? Et surtout entre linguistique populaire et linguistique dite savante ou scientifique : faut-il rester sur une opposition binaire en « vs » ou, plus raisonnablement, poser les choses en termes de continuum, de gradient de scientificité ou de spontanéité ? Les frontières sont-elles bien nettes entre purisme et linguistique populaire (le purisme, finalement, est-il aussi profane que cela) ? Et quel jeu de chaises musicales se joue entre grammaire traditionnelle, linguistique populaire et linguistique scientifique, la grammaire traditionnelle présentant, à travers la norme, des traits communs avec la linguistique populaire elle-même connectée, comme le montrent certains articles du numéro, avec la linguistique savante ? Comment définir le «populaire» ou «profane» si ce n'est d'une manière relative à un étalon de comparaison, qui serait la scientificité, elle-même susceptible de variations selon les objets, les méthodes et les objectifs considérés ?

C'est d'abord D.R. Preston, l'un des premiers défricheurs du champ et auteur avec N. Niedzielski de la première synthèse sur la folk linguistics (2003 [2000]), qui répond à ces questions en démontrant l'intérêt scientifique des commentaires linguistiques des non-linguistes. Dans l'article que le lecteur pourra consulter en ligne, il explique pourquoi et comment tenir compte de la folk linguistics, et bouscule au passage quelques idées reçues sur la question, comme la prétendue pauvreté des savoirs folk par exemple.

Plusieurs auteurs de cette livraison traitent cette dimension épistémologique : dans l'entretien qu'il nous a accordé, É. Mension-Rigau explique de manière très pratique comment il traite les données langagières, avec une méthode " bricolée » à partir de ses compétences de lecteur de littérature (c'est un historien agrégé de lettres), de ce qu'il appelle son intuition et de ses facultés d'observation et d'interprétation. Pour lui, il s'agit d'une véritable méthode historique et, comme D. Dennett, il pourrait dire qu'elle « marche », car il produit effectivement des résultats convaincants et reconnus. G. Achard-Bayle, qui a choisi de manière spéculaire une forme "populaire » pour traiter la question de la linguistique populaire (« Trivial Pursuit. Abécédaire d'identité pop-folk à l'usage des linguistes...»), montre bien comment les questions liées à la référence, au réel et à 
l'identité sont toujours susceptibles d'une théorisation à régime multiple, selon que le sens commun ou la rationalité scientifique sont avancés, l'une et l'autre étant d'ailleurs susceptibles de recevoir des définitions relatives. G. Schmale, dans «Conceptions populaires de la conversation » aborde quant à lui la question de la situation de la Laienlinguistik par rapport à d'autres pratiques, les unes moins savantes, les autres plus, à propos de la conversation. Dans une perspective épistémologique plus généraliste, M. Stegu met en regard la folk linguistics, la Laienlinguistik, la linguistique savante ou " officielle » et le rapport qu'elles entretiennent avec la linguistique appliquée. Il montre par exemple que la linguistique appliquée est à bien des égards proche de la linguistique populaire car elle a été elle-même conçue pour des non-linguistes. Il plaide donc pour une conception ouverte et scalaire des linguistiques appliquée, populaire et savante.

\subsection{Théorie}

Car, finalement, c'est la question même de la théorie que la linguistique populaire remue, en activant des interrogations sur la catégorisation et la dénomination des phénomènes étudiés en sciences du langage : l'étude des activités métalinguistiques courantes des locuteurs relève-t-elle de la linguistique populaire? Le mot courant est-il synonyme de populaire ? Et comment traiter la fameuse intuition ou le sentiment de la langue sur lesquels s'appuient les tenants d'une linguistique de la compétence ? On sait que le terme de contre-intuitif est souvent avancé pour prouver l'agrammaticalité de telle ou telle forme. L'intuition est-elle à ranger dans la catégorie du «populaire »? L'intuition est-elle profane ?

M.-A. Paveau propose quelques réponses hypothétiques à ces questions dans «Les non-linguistes font-ils de la linguistique ? Une approche anti-éliminativiste des théories folk ». Elle plaide pour une approche intégrationniste, c'est-à-dire qui intègre les données folk à la linguistique savante, sans qu'une frontière en « vs » ne soit posée entre les deux ordres de phénomènes. C'est la question de l'intuition qui permet de soutenir cette position anti-éliminativiste, dans la mesure où, la linguistique savante ne pouvant faire l'économie de l'intuition et de l'introspection, du fait de son irréductible dimension réflexive, elle présente une fragilité particulière devant les ambitions objectivistes et idéalistes issues du modèle scientifique des sciences dures. Dans le domaine de l'argumentation, M. Doury s'interroge également sur la validité des raisonnements folk. Elle montre dans son article intitulé «"Ce n'est pas un argument!" Sur quelques aspects des théorisations spontanées de l'argumentation » qu'il n'y a pas de différence criante, sur le plan de la validité des arguments, entre les productions spontanées et les productions savantes. Elle apporte ainsi de 1'eau au moulin anti-éliminativiste.

Cette position intégrationniste se retrouve même à l'intérieur de la linguistique savante elle-même, comme le montrent les travaux de F. Recanati sur le sens littéral et le sens contextuel, synthétisés dans son ouvrage Literal Meaning (Recanati 2004). Arrêtons-nous un intant sur cette conception du sens, directement liée au problème de l'éliminativisme $v s$ intégrationnisme posé dans notre numéro. F. Recanati, qui fait de la linguistique à partir de la philosophie, il faut le préciser, développe une conception contextualiste du sens, et soutient par conséquent qu'une théorie sémantique appuyée sur la notion de sens littéral ne peut fonctionner. Dans le chapitre 9 de son ouvrage, il défend une position qu'il appelle "Meaning Eliminatism » (éliminer le sens intrinsèque), reposant sur l'idée que le sens peut s'élaborer sans une conception purement linguistique et non contextuelle du sens : 
According to WF [the Wrong Format view], the sense expressed by an expression must always be contextually constructed on the basis of the (overly rich or overly abstract) meaning, or semantic potential, of the word type. [...] In that framework there still is a role for the linguistic meaning of word types : it is the input (or one of the inputs) to the construction process.

The difference between Meaning Eliminativism (ME) and WF is that, according to ME, we don't need linguistic meanings even to serve as input to the construction process. The senses that are the words' contributions to contents are constructed, but the construction can proceed without the help of conventional, context-independent word meanings (2004: 174-175).

Il considère alors que la théorie contextualiste du sens est une folk théorie dans la mesure où elle s'appuie sur des données non linguistiques, c'est-à-dire contextuelles.

Nous voilà donc devant une théorie linguistique élaborée avec une conception "non linguistique », au sens savant du terme, du sens. F. Recanati peut être décrit comme un philosophe qui fait de la linguistique avec de la non-linguistique, ce qui n'est pas du gout de tout le monde, comme le montre bien R.M. Harnish qui résume ainsi la position du philosophe français :

So Recanati seems to be not only promoting a kind of « folk semantics-pragmatics », but also denying the legitimacy (and potentiel psychological value) of traditional (" scientific ») linguistic semantics. He seems to be betting that semanticspragmatics will not go the way of syntax (and phonology). That is, that introspective, folk psychology will trump semantic intutitions plus the traditional methodology of theory construction in semantics (Harnish $2005: 397$ ).

Toute linguistique ne serait-elle pas une folk linguistique ? C'est ce que montrent en tout cas certaines représentations discursives et certains imaginaires linguistiques.

\subsection{Représentations}

La question de la validité théorique ou plus largement scientifique se pose également pour les études sur les représentations linguistiques, de l'imaginaire linguistique formulé par A.-M. Houdebine aux mythologies linguistiques étudiées par P. Zoberman, en passant par les linguistiques fantastiques et imaginaires évoquées par M. Yaguello : l'imaginaire est-il populaire, la linguistique fantastique est-elle profane ? De quels domaines, de quelles catégories s'agit-il, dans quel champ disciplinaire ou sous-disciplinaire sommes-nous?

Des réponses sont apportées par J.-C. Beacco qui se propose d'analyser « les conceptions circulantes de ce que sont le bien parler ou le bien écrire authentiques, qui répondent à des valeurs distinctes de ou opposées à celles qui fondent les normes dominantes dites, indifféremment, académiques, officielles, scolaires, surveillées, élégantes...». Dans son article intitulé « De la verve. À la recherche d'un idéal discursif ordinaire », il examine un corpus d'articles de la presse sportive (le quotidien L'Équipe) pour débusquer les définitions spontanées de ce que serait cette verve authentique. Dans un autre ordre d'idées mais toujours dans le domaine des cadres représentationnels, P. Sériot, E. Bulgakova et A. Heržen montrent dans « La linguistique populaire et les pseudo-savants », que le discours sur la langue constitue le fondement d'un questionnement sur les identités collectives et nationales en Europe de 1'Est actuellement. Ils mettent 
ainsi en exergue des réalités culturelles et discursives peu connues, comme la fabrication d'identités mythiques et d'origines fantastiques par le biais d'un discours linguistique "pseudo-savant» à forte intention persuasive. Les enjeux sont de taille puisqu'il en va de la définition des peuples et de la délimitation des frontières.

\subsection{Pratiques}

La linguistique populaire pose enfin le problème de la valeur et de l'efficacité des savoirs spontanés en contexte d'apprentissage : un discours fréquent est que, en linguistique comme ailleurs, la démonstration scientifique contredit le plus souvent l'interprétation spontanée (c'est par exemple la position de Jackendoff 2003 par rapport au «folk knowledge»). Ce discours est cependant battu en brèche actuellement par certains philosophes des sciences qui montrent, après d'illustres prédécesseurs comme A. Schutz ou P. Feyerabend, à quel point les théories spontanées sont parfois proches des résultats scientifiques, ou possèdent une validité d'un autre ordre nécessaire dans le champ de la vie en société.

Cette question nous semble cruciale en classe de français de la maternelle à l'université. Quelle est la place des savoirs profanes des élèves et des enseignants en classe (on sait que l'activité métalinguistique des « enfants grammairiens » est largement exploitée dans les pré-apprentissages à la maternelle et au cycle 1) ? Une " erreur scientifique » peut avoir une bonne rentabilité fonctionnelle dans l'apprentissage ? Est-ce le cas pour toutes les activités ? Pour tous les niveaux d'enseignement (« l'enfant grammairien » de Gleitman (1972) peut-il devenir un « étudiant grammairien ») ? L'enseignement du français doit-il privilégier efficacité ou exactitude scientifique?

Les auteurs de la troisième partie de ce numéro apportent des réponses qui concernent l'orthographe et les graphies, les raisonnements sur la langue et la grammaire.

Dans son travail sur « Les auto-explications métagraphiques appliquées aux premières écritures enfantines », J. David s'appuie sur un large corpus de textes écrits et surtout d'auto-explications ou commentaires métagraphiques produits par de jeunes enfants apprentis-scripteurs (5-7 ans) pour montrer comment ils parviennent à verbaliser des savoirs émergents sur le fonctionnement de l'écrit. Ce savoir s'avère présenter des connexions, d'une part avec les propriétés des langues orales, et d'autre part avec les composantes de l'écriture (sémiologiques, phonologiques, morphologiques) progressivement découvertes et intégrées. Un savoir spontané incontestable sort donc des commentaires métagraphiques des enfants. Des constatations analogues sont faites par à propos des écritures inventées (invented spellings) par J.-P. Jaffré et M.-F. Morin. Dans leur réflexion sur "Les activités pré-orthographiques : nature, validité et conceptions », ils montrent en effet que les jeunes enfants détiennent des compétences pré-orthographiques car ils ne sont pas d'abord cognitivement sensibles à la norme orthographique mais à des fonctionnements sous-jacents dont seule la prise en compte permet d'évaluer leurs productions précoces. Ils en concluent que, écrire, ce n'est plus alors (re)produire de la norme orthographique mais percevoir des principes graphiques élémentaires et récurrents - comme par exemple la valeur phonologique de lettres de la tradition alphabétique.

Ce sont ces « enfants grammairiens » qui font l'objet des commentaires de M. Laparra. Dans «L'élève grammairien ? L'exemple de l'apprentissage des 
marques graphiques du français », elle se demande quelle est la valeur des savoirs spontanés que les élèves peuvent produire sur certaines marques morphologiques graphiques du français. La classe reste malgré tout un espace interactionnel qui constitue un véritable réservoir où l'on repère des discours tantôt préfabriqués, furtifs ou ritualisés, tantôt issus de savoirs scolaires eux-mêmes dérivés des savoirs savants. C'est ce que montre C. Weber dans « Les verbalisations ordinaires dans la classe : objets furtifs ou variables encombrantes des sciences du langage ? ». Les modes communicationnels variés qui se jouent dans la classe donnent lieu en effet selon elle à une infinie variation de productions, de représentations et de raisonnements en voie de se construire, dont la forme et la présence sont « furtives », mais qui interviennent de façon décisive dans les pratiques scolaires.

L'entretien avec notre folk linguiste-historien É. Mension-Rigau constituait une ouverture initiale à l'ensemble des travaux rassemblés dans cette livraison. L'article de F. Capucho leur propose ce que nous avons appelé une ouverture finale. Elle propose en effet une approche inédite dans le numéro dans la mesure où elle choisit de travailler dans le contexte du plurilinguisme, et sur une notion à dimension pluridisciplinaire (linguistique, philosophie, psychologie sociale, communication), l'intercompréhension. Dans « L'intercompréhension est-elle une mode ? Du linguiste citoyen au citoyen plurilingue », elle montre que la notion structure les comportements des locuteurs plurilingues, en particulier dans des conditions à fort coefficient d'étrangéité comme les voyages. Elle analyse en particulier les composantes émotionnelle et situationnelle et montre à quel point les locuteurs mobilisent spontanément, presque " naturellement » leur compétence intercompréhensive.

Nous souhaitons que ce numéro balise et éclaire un domaine encore un peu obscur en France, de manière à permettre des interrogations d'ordre épistémologique, théorique et pratique qui nous semblent nécessaires pour que le champ des sciences du langage se maintienne dans un dynamisme assuré. Nous espérons que les pistes ouvertes ou approfondies ici favoriseront également les collaborations européennes et internationales sur la question de la linguistique populaire et plus généralement de la validité des savoirs issus des sciences humaines. Les chercheurs rassemblés ici représentent la linguistique française, suisse, allemande, autrichienne, portugaise, canadienne, américaine ; ils constituent de fait un réseau de savoir international dont nous souhaitons le développement dynamique et collaboratif.

Guy Achard-Bayle

Marie-Anne Paveau 


\section{Références}

AuthiER-REVUZ, J. (1995) : Ces mots qui ne vont pas de soi. Boucles réflexives et non-coïncidences $d u$ dire, Paris, Larousse, 2 tomes.

Antos, G. (1996) : Laien-Linguistik. Studien zu Sprach-und Kommunikationsproblemen im Alltag, Tübingen, Niemeyer.

BEACCO, J.-C. (dir.) (2004) : «Représentations métalinguistiques ordinaires et discours », Langages 154, Paris, Larousse.

BERRENDONNER, A. (1982) : L'éternel grammairien. Étude du discours normatif, Berne, Peter Lang.

BOUCHARD, R., MEYER, J.-C. (1995) : Les métalangages de la classe de français, s.1., DFLM.

BourdiEU, P. (2001) : « Vous avez dit “populaire” ? », dans Langage et pouvoir symbolique, Paris, Seuil : 132-151.

BREKLE, H.E. (1989) : «La linguistique populaire», dans Auroux, S. (dir.), Histoire des idées linguistiques, Bruxelles, Mardaga, tome 1 : 39-44.

Contes, J. (1996) : The Claim of Commonsense. Moore, Wittgenstein and the Social Sciences, Cambridge, Cambridge University Press.

D ASCAL, M. (1999) : «Trois préjugés sur le préjugé », dans Amossy, R. et Delon, M. (dir.), Critique et légitimité du préjugé, du XVIIè siècle à nos jours, Bruxelles, Presses Universitaires de Bruxelles : 113-118.

DennetT, D. (1987) : The Intentional Stance, trad. franç. P. Engel, 1990, La Stratégie de l'interprète. Le sens commun et l'univers quotidien, Paris, Gallimard, «NRF essais ».

Fisette, D., POIRIER, P. (éds.) (2002) : Philosophie de l'esprit. Psychologie du sens commun et sciences de l'esprit, Paris, Vrin.

FREI, H. (1982 [1928]) : La Grammaire des fautes, Genève-Paris, Slatkine reprints.

GADET, F. (2007 [2003]) : La variation sociale en français, Paris, Ophrys.

Gleitman, L., Gleitman, H., Shipley, E. (1972) : « The Emergence of the Child as a Grammarian », Cognition 1-2/3 : 137-164.

HARNISH, R.M. (2005) : «Folk psychology and literal meaning », Pragmatics and cognition 13.2 : 382-399.

Heintz, C., Pouscoulous, N. \& TARABorelli, D. (eds.) (2008) : « Folk Epistemology », European Review of Philosophy, 8.

Hoenigswald, H. M. (1960) : Language Change and Linguistic Reconstruction, Chicago, University of Chicago Press.

— (1966) : «A Proposal for the Study of Folk-linguistics », in Sociolinguistics : Proceedings of the UCLA Sociolinguistic Conference 1964, ed. by W. Bright, The Hague, Mouton : 16-26.

Houdebine, A.-M. (2002) : L'imaginaire linguistique, Paris : 1'Harmattan.

JACKENDOFF, R. (2003) : «The Structure of Language : Why It Matters to Education ", Talk for conference on Learning and the Brain, Linguistic Society of America, Cambridge, November 5-8, www.edupr.com.

Julia, C. (2001) : Fixer le sens? La sémantique spontanée des gloses de spécification du sens, Paris, PSN.

LANGer, N. (2001) : « Linguistic Purism in Action - How auxiliary “tun” was stigmatized in Early New High German », Studia Linguistica Germania 60, Berlin, New York : de Gruyter. 
LAFONTAINE, D. (1986) : Le parti pris des mots. Normes et attitudes linguistiques, Bruxelles, Mardaga.

LEEMAN-BouiX, D. (1994) : Les fautes de français existent-elles?, Paris, Seuil.

MARKOVITS, F. (1999) : «Histoire naturelle du préjugé » dans AMOSSY \& DELON (dir.), , Critique et légitimité du préjugé, du XVIIè siècle à nos jours, Bruxelles, Presses Universitaires de Bruxelles : 73-90.

NiedZIELSKI, N. \& PRESTON, D. (2003 [2000]) : [with a new preface], Folk Linguistics, Berlin, New York, Mouton De Gruyter.

PAVEAU, M.-A. (2000) : «La "richesse lexicale", entre apprentissage et acculturation », Le Français aujourd'hui 131, "Construire les compétences lexicales », Paris, AFEF : 19-30.

- (2005) : «Linguistique populaire et enseignement de la langue : des catégories communes? », Le Français aujourd'hui 151, Paris AFEF-Armand Colin : 95-107.

- (2006) : Les prédiscours. Sens, mémoire, cognition, Paris, Presses Sorbonne nouvelle.

- (2007) : «Les normes perceptives de la linguistique populaire », Langage et société 121, «Les normes pratiques » : 93-109.

— (2008) : «Le parler des classes dominantes, objet linguistiquement incorrect? Dialectologie perceptive et linguistique populaire », Études de linguistique appliquée 150 : 137-156.

PaVeau, M.-A., Rosier, L. (2008) : La langue française. Passions et polémiques, Paris, Vuibert.

PHILIPPE, G. (2002) : Sujet, verbe, complément. Le moment grammatical de la littérature française 1890-1940, Paris, Gallimard.

REY-DEBOVE, J. (1978) : Le métalangage. Étude linguistique du discours sur le langage, Paris, Le Robert.

ROSIER, L. (2003) : «Du discours rapporté à la circulation des discours : 1'exemple des dictionnaires de "critique ironique" ", Estudios de lengua y literatura francesas 14, Cadiz, Universidad de Cadiz : 63-81.

— (2004) : « La circulation des discours à la lumière de "l'effacement énonciatif" : 1'exemple du discours puriste sur la langue », Langages 156, Paris, Larousse : 65-78.

- (2006) : Petit traité de l'insulte, Bruxelles, Labor.

SÉRIOT, P. (1996) : «La linguistique spontanée des traceurs de frontières », in Sériot P. (éd.), Langue et nation en Europe centrale et orientale du 18 ème siècle à nos jours, Cahiers de l'ILSL 8, Lausanne : 277-304. 